\title{
Simulation and construction of single phase flying capacitor multilevel inverter
}

\begin{abstract}
This paper focuses on the simulation and construction of single phase, three level Flying Capacitor Multilevel Inverter (FCMI). For FCMI, there is several switching state for one DC level of the inverter, so the selection of switching state combination is important so that every IGBTs can be switched on equally. The design of the FCMI was successful done on the MATLAB/simulink simulation software including the triggering circuit. The small scale laboratory model of FCMI was also successfully constructed. A good agreement between simulation and hardware results has been obtained.
\end{abstract}

Keyword: Flying capacitor; Multilevel inverter 